\title{
Berenil Binds Tightly to Parallel and Mixed Parallel/Antiparallel G-Quadruplex Motifs with Varied Thermodynamic Signatures
}

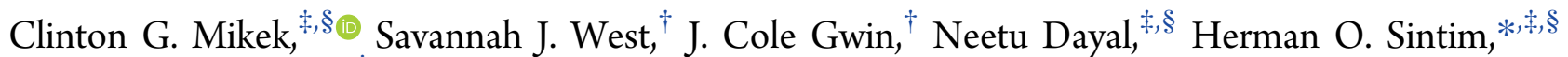
and Edwin A. Lewis* ${ }^{*} \dagger$ (이

\author{
${ }^{\dagger}$ Department of Chemistry, Mississippi State University, 310 President’s Circle, Mississippi, Mississippi State 39762, United States \\ ${ }^{\ddagger}$ Department of Chemistry, Purdue University, 560 Oval Drive, West Lafayette, Indiana 47907, United States \\ ${ }^{\S}$ Center for Drug Discovery, Purdue University, 720 Clinic Drive, West Lafayette, Indiana 47907, United States
}

\author{
Supporting Information
}

\begin{abstract}
Diminazene, DMZ, (or berenil) has been reported as a tight binder of G-quadruplexes. G-Quadruplex structures are often located in the promotor regions of oncogenes and may play a regulatory role in gene expression based on the stability of the folding topology. In this study, attempts have been made to characterize the specificity of DMZ binding toward multiple G-quadruplex topologies or foldamers. Mutant sequences of the G-quadruplex forming promotor regions of several oncogenes were designed to exhibit restricted loop lengths and folding topologies. Circular dichroism was used to confirm the quadruplex topology of mutant BCL2, KRAS, and c-MYC sequences, human telomere $\left(\mathrm{Na}^{+}\right.$and $\left.\mathrm{K}^{+}\right) \mathrm{G}$-quadruplexes and their complexes with DMZ

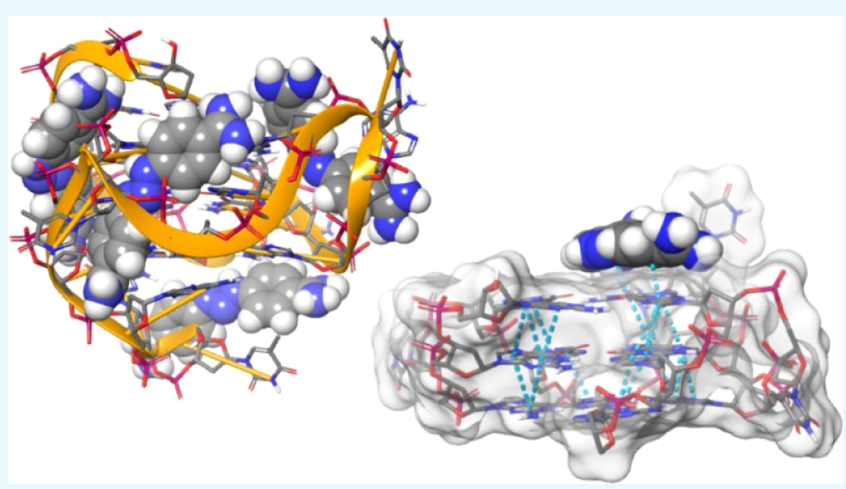
and analogs thereof. Isothermal titration calorimetry was used to generate a complete thermodynamic profile $(\Delta G, \Delta H,-T \Delta S)$ for the formation of DMZ and analog complexes with the target G-quadruplexes. DMZ binds to parallel and/or mixed parallel/ antiparallel quadruplex DNA motifs with stoichiometries up to 8:1 and via three binding modes with varying affinities. In the case of the parallel G-quadruplexes, with the exception of the long-looped c-MYC mutant, the highest affinity binding event (mode 1) is driven by enthalpy. DMZ binding to the long-looped c-MYC mutant exhibits a very favorable entropy change in addition to a moderately favorable enthalpy change. Mode 1 binding to the antiparallel and mixed parallel/antiparallel hTel quadruplexes is also driven by favorable enthalpy changes. In all cases, the intermediate DMZ affinity binding (mode 2) is driven almost entirely by entropy, with small or unfavorable enthalpic contributions. The weakest binding event (mode 3 ) is also entropically driven with small or moderate enthalpic contributions.
\end{abstract}

\section{INTRODUCTION}

Both DNA and RNA can form many secondary structures, such as G-quadruplex (G4), ${ }^{1}$ triplex, ${ }^{2}$ i-motif, ${ }^{3}$ and a biological role for these structures, especially G4s, ${ }^{4}$ has been suggested. There are approximately 3.8 million guanine-rich regions in the human genome, which have G4 forming potential, ${ }^{5}$ including those located near the telomeric ${ }^{6}$ and promoter regions of some cancer-related genes. ${ }^{4 a}$

G4 motifs located in the promotor region of certain genes must be unfolded for transcription to occur as observed in oncogenes, including c-MYC, ${ }^{7}$ KRAS, ${ }^{8}$ and BCL2. ${ }^{9}$ Small molecules that target and stabilize these structures could have therapeutic value. Transcription of oncogenes, including c$\mathrm{MYC}^{10} \mathrm{c}-\mathrm{Kit},{ }^{11}$ and $\mathrm{KRAS},{ }^{8}$ has been down regulated via ligand-induced stabilization of the G4, and thus these types of compounds may have a potential use as antineoplastics in cancer lines with G4-related drivers. ${ }^{12}$ G4 stabilizing ligands, such as pyridostatin, Phen-DC3, and 12459, have been shown to reduce the activity of a G4 unfolding enzyme by stabilizing the G4. ${ }^{13}$
Due to the expected important biological role the G4 motif has in modulating oncogene expression, there is interest in identifying G4 selective ligands for fundamental studies (for example, fluorescent ligands allowing the study of G4s in vivo) and also druglike molecules that will allow for selective targeting of G4s related to cancer ${ }^{14}$ and other diseases. ${ }^{15}$ Recently, Marchetti et al. demonstrated the transcriptional down regulation of genes in a human pancreatic ductal adenocarcinoma model connected to survival, metastasis, and drug resistance. ${ }^{16}$ The authors rationally designed a G4-interactive ligand and used global genome transcriptome profiling to identify targets of G4-binding molecules in two pancreatic cancer cell lines (MIA PaCa-2 and PANC-1). ${ }^{16}$ The lead compound CM03 exhibited mRNA down regulation in cellulo for genes identified in the genome profiling and tumor growth

Received: July 11, 2018

Accepted: September 6, 2018

Published: September 21, 2018 
Table 1. Thermodynamic Parameters for c-MYC Mutant Titrations by DMZ in $\mathrm{K}^{+}$Tris Buffer $(150 \mathrm{mM} \mathrm{KCl,} 20 \mathrm{mM}$ Tris, $1 \mathrm{mM}$ Ethylenediaminetetraacetic Acid (EDTA), 30\% Dimethyl Sulfoxide (DMSO), pH 7.2 ${ }^{a}$

\begin{tabular}{|c|c|c|c|c|c|}
\hline parallel & c-MYC 1-6-1 & $K_{\mathrm{a}}\left(\times 10^{7}\right)$ & $\Delta G\left(\mathrm{kcal} \mathrm{mol}^{-1}\right)$ & $\Delta H\left(\mathrm{kcal} \mathrm{mol}^{-1}\right)$ & $-T \Delta S\left(\mathrm{kcal} \mathrm{mol}^{-1}\right)$ \\
\hline & mode 1 & 8.13 & $-10.77 \pm 0.15$ & $-3.38 \pm 0.63$ & $-7.39 \pm 0.62$ \\
\hline & mode 2 & 0.557 & $-9.18 \pm 0.16$ & $-0.31 \pm 0.04$ & $-8.89 \pm 0.12$ \\
\hline & mode 3 & 0.00300 & $-6.08 \pm 0.22$ & $-1.46 \pm 0.15$ & $-4.62 \pm 0.36$ \\
\hline \multirow[t]{4}{*}{ parallel } & c-MYC 1-2-1 & $K_{\mathrm{a}}\left(\times 10^{7}\right)$ & $\Delta G$ & $\Delta H$ & $-T \Delta S$ \\
\hline & mode 1 & 23.0 & $-11.37 \pm 0.32$ & $-8.38 \pm 0.88$ & $-2.97 \pm 0.82$ \\
\hline & mode 2 & 1.87 & $-9.84 \pm 0.37$ & $-0.11 \pm 0.07$ & $-9.73 \pm 0.39$ \\
\hline & mode 3 & 0.00587 & $-6.43 \pm 0.33$ & $-1.27 \pm 0.35$ & $-5.16 \pm 0.68$ \\
\hline \multirow[t]{4}{*}{ parallel } & BCL2 3-7-1 & $K_{\mathrm{a}}\left(\times 10^{7}\right)$ & $\Delta G$ & $\Delta H$ & $-T \Delta S$ \\
\hline & mode 1 & 22.0 & $-11.35 \pm 0.21$ & $-6.58 \pm 0.14$ & $-4.78 \pm 0.37$ \\
\hline & mode 2 & 1.55 & $-9.79 \pm 0.20$ & $-0.29 \pm 0.03$ & $-9.50 \pm 0.17$ \\
\hline & mode 3 & 0.00455 & $-6.35 \pm 0.11$ & $-2.06 \pm 0.06$ & $-4.29 \pm 0.17$ \\
\hline \multirow[t]{4}{*}{ parallel } & BCL2 3-6-2 & $K_{\mathrm{a}}\left(\times 10^{7}\right)$ & $\Delta G$ & $\Delta H$ & $-T \Delta S$ \\
\hline & mode 1 & 41.5 & $-11.75 \pm 0.07$ & $-8.77 \pm 0.08$ & $-2.98 \pm 0.12$ \\
\hline & mode 2 & 3.40 & $-10.30 \pm 0.00$ & $-0.18 \pm 0.01$ & $-10.09 \pm 0.02$ \\
\hline & mode 3 & 0.00690 & $-6.60 \pm 0.01$ & $-1.15 \pm 0.00$ & $-5.44 \pm 0.01$ \\
\hline \multirow{4}{*}{ mixed } & BCL2 3-5-3 & $K_{\mathrm{a}}\left(\times 10^{7}\right)$ & $\Delta G$ & $\Delta H$ & $-T \Delta S$ \\
\hline & mode 1 & 17.5 & $-11.20 \pm 0.14$ & $-2.08 \pm 0.37$ & $-9.15 \pm 0.54$ \\
\hline & mode 2 & 0.715 & $-9.34 \pm 0.16$ & $-0.23 \pm 0.04$ & $-8.95 \pm 0.21$ \\
\hline & mode 3 & 0.00190 & $-5.83 \pm 0.08$ & $-0.84 \pm 0.15$ & $-4.99 \pm 0.24$ \\
\hline \multirow[t]{4}{*}{ mixed } & KRAS 6-4-4 & $K_{\mathrm{a}}\left(\times 10^{7}\right)$ & $\Delta G$ & $\Delta H$ & $-T \Delta S$ \\
\hline & mode 1 & 25.5 & $-11.45 \pm 0.07$ & $-4.30 \pm 0.00$ & $-7.16 \pm 0.01$ \\
\hline & mode 2 & 1.60 & $-9.82 \pm 0.11$ & $-0.15 \pm 0.04$ & $-9.67 \pm 0.07$ \\
\hline & mode 3 & 0.00210 & $-5.88 \pm 0.16$ & $-0.45 \pm 0.04$ & $-5.43 \pm 0.20$ \\
\hline \multirow[t]{4}{*}{ mixed } & KRAS 6-4-5 & $K_{\mathrm{a}}\left(\times 10^{7}\right)$ & $\Delta G$ & $\Delta H$ & $-T \Delta S$ \\
\hline & mode 1 & 5.90 & $-10.60 \pm 0.00$ & $-3.41 \pm 0.10$ & $-7.19 \pm 0.12$ \\
\hline & mode 2 & 0.270 & $-8.77 \pm 0.01$ & $-0.36 \pm 0.09$ & $-8.41 \pm 0.09$ \\
\hline & mode 3 & 0.000865 & $-5.37 \pm 0.11$ & $-1.24 \pm 0.17$ & $-4.12 \pm 0.28$ \\
\hline \multirow[t]{4}{*}{ mixed } & KRAS 6-4-6 & $K_{\mathrm{a}}\left(\times 10^{7}\right)$ & $\Delta G$ & $\Delta H$ & $-T \Delta S$ \\
\hline & mode 1 & 3.85 & $-10.30 \pm 0.01$ & $-8.33 \pm 0.12$ & $-2.02 \pm 0.13$ \\
\hline & mode 2 & 0.415 & $-9.02 \pm 0.03$ & $-0.43 \pm 0.04$ & $-8.59 \pm 0.07$ \\
\hline & mode 3 & 0.000970 & $-5.44 \pm 0.13$ & $-1.37 \pm 0.01$ & $-4.07 \pm 0.11$ \\
\hline \multirow[t]{4}{*}{ mixed } & hTel22 $\mathrm{K}^{+}$ & $K_{\mathrm{a}}\left(\times 10^{7}\right)$ & $\Delta G$ & $\Delta H$ & $-T \Delta S$ \\
\hline & mode 1 & 13.7 & $-11.07 \pm 0.1$ & $-8.43 \pm 0.7$ & $-2.7 \pm 0.7$ \\
\hline & mode 2 & 1.40 & $-9.7 \pm 0.1$ & $-0.06 \pm 0.04$ & $-9.7 \pm 0.2$ \\
\hline & mode 3 & 0.00623 & $-6.53 \pm 0.08$ & $-0.42 \pm 0.08$ & $-6.1 \pm 0.2$ \\
\hline \multirow[t]{4}{*}{$\begin{array}{c}\text { anti- } \\
\text { parallel }\end{array}$} & ${ }^{*} \mathrm{hTel22} \mathrm{Na}^{+}$ & $K_{\mathrm{a}}\left(\times 10^{7}\right)$ & $\Delta G$ & $\Delta H$ & $-T \Delta S$ \\
\hline & mode 1 & 18.2 & $-11.2 \pm 0.4$ & $-10.4 \pm 0.9$ & $-0.81 \pm 1.28$ \\
\hline & mode 2 & 4.67 & $-10.38 \pm 0.4$ & $0.18 \pm 0.07$ & $-10.56 \pm 0.33$ \\
\hline & mode 3 & 0.0240 & $-7.33 \pm 0.11$ & $-0.33 \pm 0.02$ & $-7.01 \pm 0.13$ \\
\hline
\end{tabular}

inhibition was observed in a pancreatic ductal adenocarcinoma animal model. ${ }^{16}$

Efforts have been underway to create high affinity and selective G4 binders using several approaches, including the use of multicarbazole scaffolds, ${ }^{17}$ metallopeptides, ${ }^{18}$ tetracyclic anthraquinone derivatives, ${ }^{19}$ and natural compound derivatives $^{20}$ to name a few. G4-DNA has emerged as a potentially relevant clinical target. ${ }^{21}$ Compounds that selectively bind individual G4 present in promotor regions of oncogenes may become useful for targeted treatments. Since individual G4 sequences tend to fold into a major population, identifying compounds that selectively stabilize individual topologies is an attractive prospect to target G4 regulated genes. Mutant G4s with restricted folding topologies limit the foldamers available by modulating the number and length of G-repeats. The repurposing of drugs used in human and veterinary medicine for anticancer therapies seems logical due to the potential translational potential of such drugs or analogs since the molecules are already in clinical use. ${ }^{22}$ The diminazene (DMZ or berenil) scaffold was recently built upon to form alykynesubstituted DMZ analogs as G4 binders. ${ }^{22}$ The lead DMZ analogs were shown to be able to target c-MYC G4 both in vitro and in cellulo and display reasonable anticancer activities (single-digit micromolar $\mathrm{GI}_{50}$ ) against ovarian (OVCAR-3), prostate (PC-3), and triple negative breast (MDA-MB-231) cancer cell lines. $^{22}$

Along the lines of the previously reported high affinity for several quadruplex structures, ${ }^{23}$ the DMZ scaffold is an attractive starting point to develop potent G4 ligands. Consequently, we sought to revisit the characterization of 

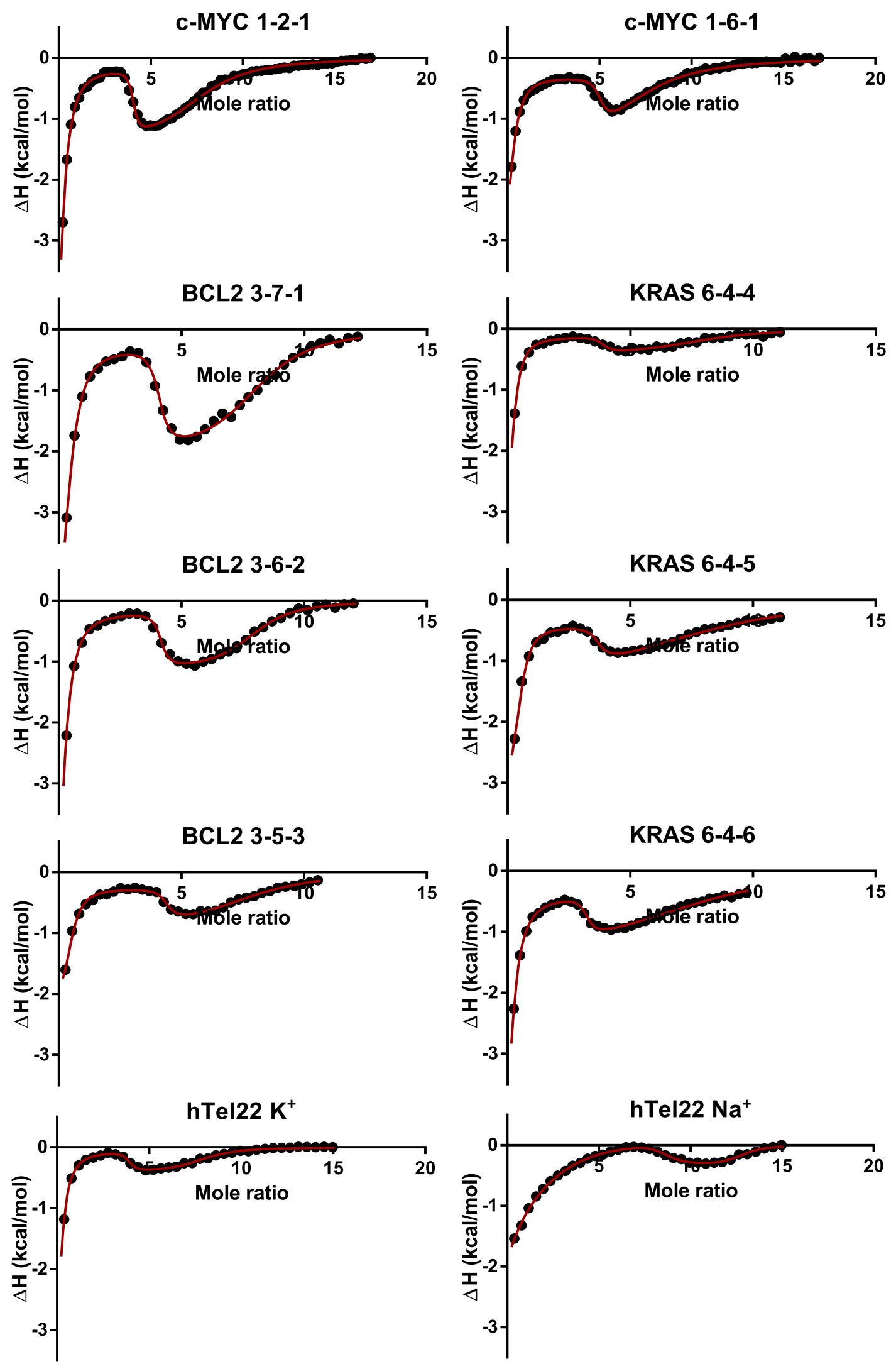

Figure 1. ITC thermograms and nonlinear regression fit lines for the titration of DMZ into the indicated G4 forming oligonucleotide.

DMZ binding to several oncogene promotor G4-DNA mutants with restricted folding topologies. In this work, DMZ binding was examined, and analogs were made by modifying the amidine groups, cyclizing, and otherwise modifying the triazene region of the DMZ scaffold. 
c-MYC 1-6-1

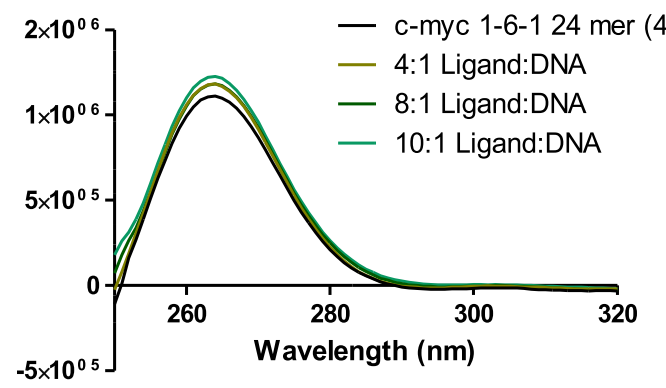

BCL2 3-5-3
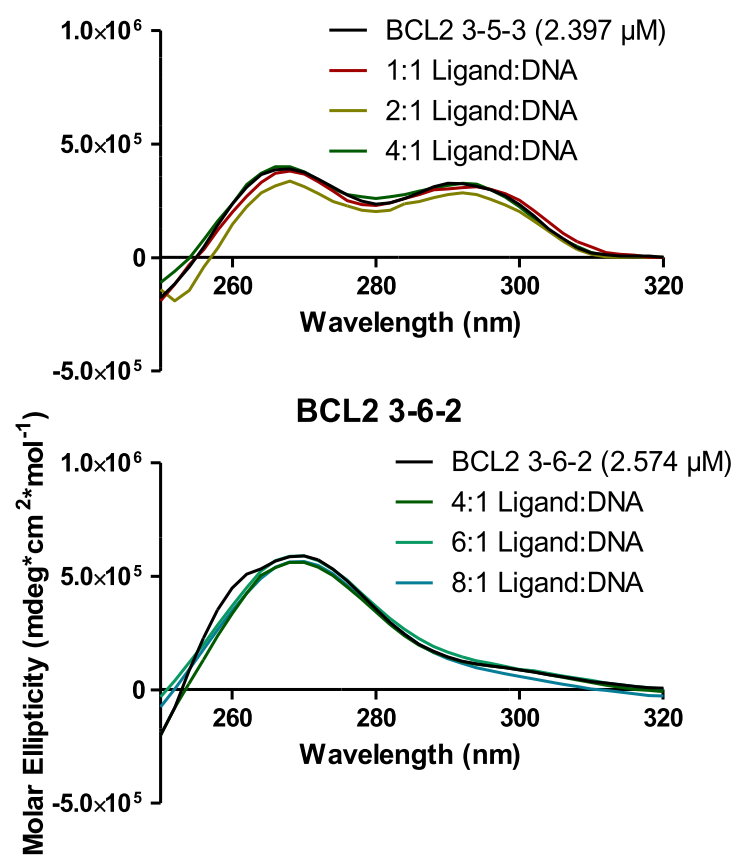

BCL2 3-7-1

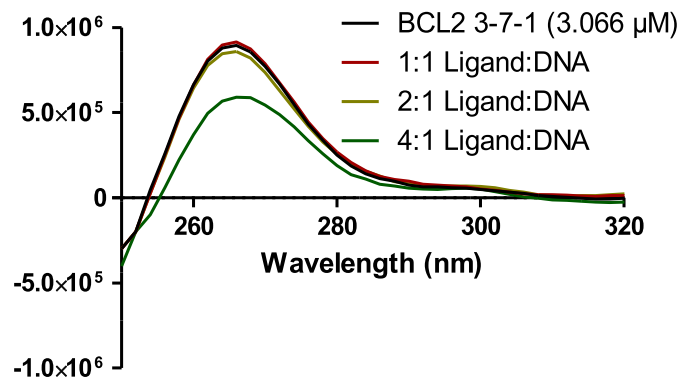

hTel22 Na+

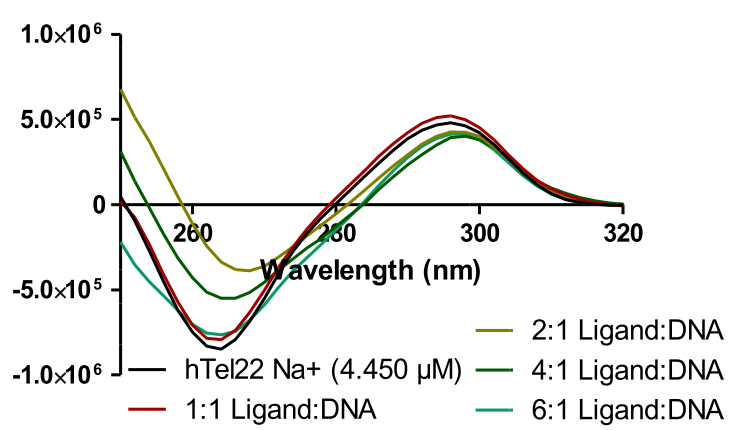

c-MYC 1-2-1

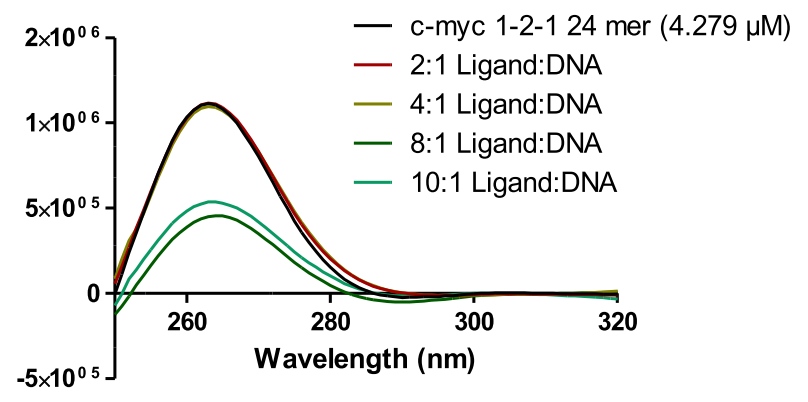

KRAS 6-4-6

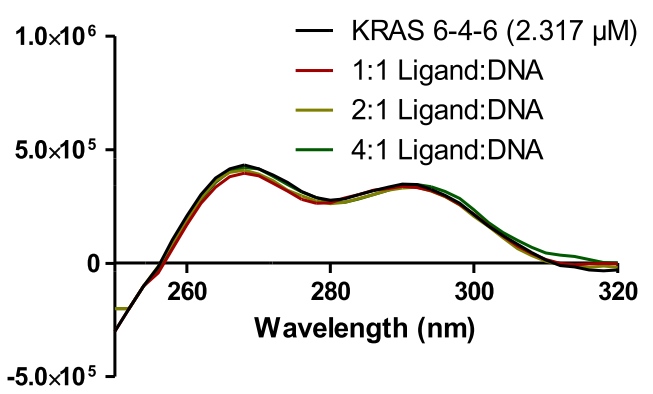

KRAS 6-4-5

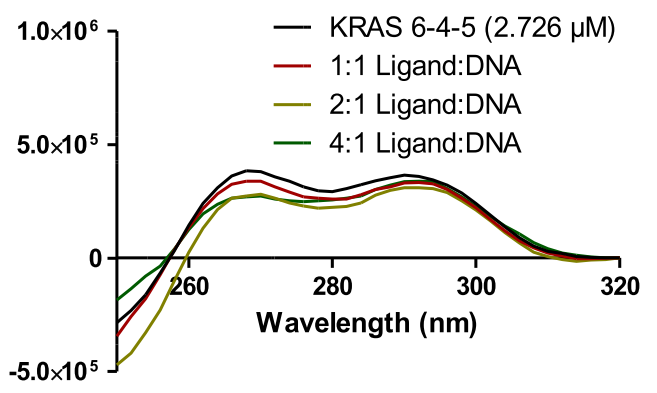

KRAS 6-4-4
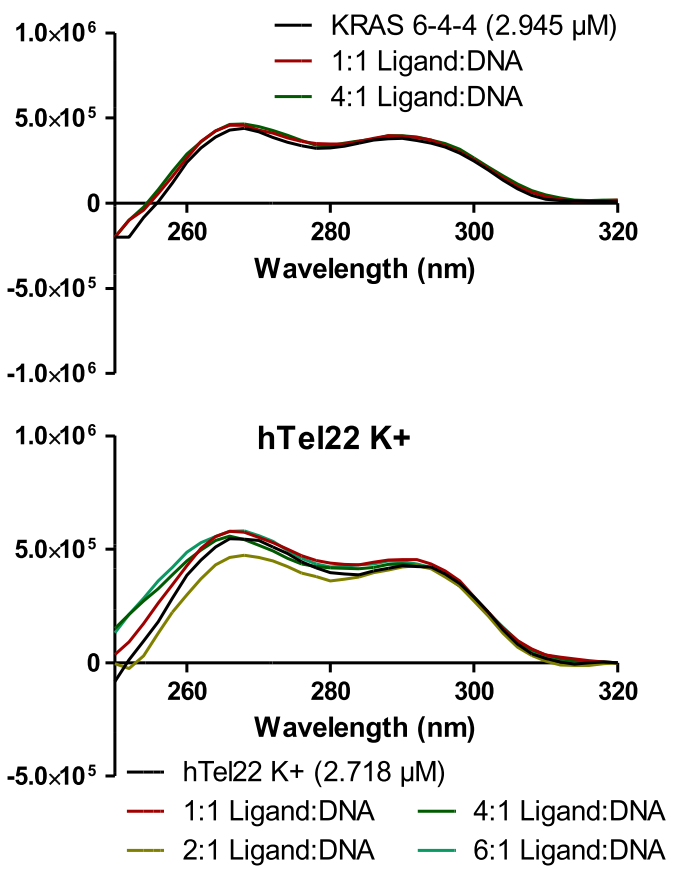

Figure 2. Circular dichroism data for the titration of BCL2, KRAS, and c-MYC G4 forming mutants titrated with DMZ. 


\section{RESULTS AND DISCUSSION}

Isothermal Titration Calorimetry (ITC). As is often the case, the endpoints in ITC titrations with multiple overlapping equilibria are difficult to determine with a high degree of certainty. For this reason, we fixed the total ligand (DMZ analog) to G4 stoichiometry at eight $(8: 1)$. This value for $n_{\text {total }}$ (8:1) is consistent with the analytical data, e.g., the electrospray ionisation mass spectrometry (ESI-MS) ${ }^{23}$ data as well as the circular dichroism (CD) and ITC saturation endpoint data. Some of the ITC thermograms required three equilibria to be fit within experimental error, whereas a few only required two equilibria. The nominal values of $n_{i}$ for the three equilibria case were determined to be $n_{1}=0.25-0.5 ; n_{2}=3.5-3.75$; and $n_{3} \approx 4$, whereas for the two equilibria case the values of $n_{1}$ and $n_{2}$ were both nominally equal to 4 . The distinction between the two cases is whether the highest affinity binding process is described by one or two independent binding events. Reducing the number of fitting parameters from nine $\left(K_{1}, K_{2}, K_{3}, \Delta H_{1}, \Delta H_{2}\right.$, $\Delta H_{3}, n_{1}, n_{2}$, and $\left.n_{3}\right)$ to six $\left(K_{1}, K_{2}, K_{3}, \Delta H_{1}, \Delta H_{2}, \Delta H_{3}\right)$ results in better fits without the loss of important thermodynamic information (Table 1, Figure 1).

Mode 1. The change in Gibbs free energy $(\Delta G)$ upon complexation of DMZ to G4-DNA differed by only $1.45 \mathrm{kcal}$ $\mathrm{mol}^{-1}$ and ranged from $-11.75 \mathrm{kcal} \mathrm{mol}^{-1}$ (BCL2 3-6-2) to $-10.3 \mathrm{kcal} \mathrm{mol}^{-1}$ (KRAS 6-4-6) for binding mode 1. Despite this small change in free energy, the enthalpic term $(\Delta H)$ ranges from $-10.36 \mathrm{kcal} \mathrm{mol}^{-1}\left(\mathrm{hTel} 22 \mathrm{Na}^{+}\right)$to $-2.08 \mathrm{kcal} \mathrm{mol}^{-1}$ (BCL2 3-5-3) for binding mode 1, differing by $8.28 \mathrm{kcal} \mathrm{mol}^{-1}$. Entropic contributions $(-T \Delta S)$ range from $-9.15 \mathrm{kcal} \mathrm{mol}^{-1}$ (BCL2 3-5-3) to $-2.02 \mathrm{kcal} \mathrm{mol}^{-1}$ (KRAS 6-4-6) for binding mode 1 , which is a difference of $8.34 \mathrm{kcal} \mathrm{mol}^{-1}$.

Mode 2. The change in Gibbs free energy $(\Delta G)$ upon complexation of DMZ to G4-DNA ranges from $-10.38 \mathrm{kcal}$ $\mathrm{mol}^{-1}\left(\mathrm{hTel22} \mathrm{Na}^{+}\right.$) to $-8.77 \mathrm{kcal} \mathrm{mol}^{-1}$ (KRAS 6-4-5) for binding mode 2, which is a difference of $1.61 \mathrm{kcal} \mathrm{mol}^{-1}$. The enthalpy change $(\Delta H)$ ranged from $-0.43 \mathrm{kcal} \mathrm{mol}^{-1}$ (KRAS 64-6) to $0.18 \mathrm{kcal} \mathrm{mol}^{-1}\left(\mathrm{hTel} 22 \mathrm{Na}^{+}\right)$for binding mode 2, which is a difference of only $0.61 \mathrm{kcal} \mathrm{mol}^{-1}$. The entropy contribution $(-T \Delta S)$ ranges from $-10.56 \mathrm{kcal} \mathrm{mol}^{-1}\left(\mathrm{hTel} 22 \mathrm{Na}^{+}\right)$to -8.41 kcal mol ${ }^{-1}$ (KRAS 6-4-5) for binding mode 2 yielding a difference of $2.15 \mathrm{kcal} \mathrm{mol}^{-1}$.

Mode 3. The change in Gibbs free energy $(\Delta G)$ upon complexation of DMZ to G4-DNA ranges from $-7.33 \mathrm{kcal}$ $\mathrm{mol}^{-1}\left(\mathrm{hTel22} \mathrm{Na}{ }^{+}\right.$) to $-5.37 \mathrm{kcal} \mathrm{mol}^{-1}$ (KRAS 6-4-5) for binding mode 3 , which had a range of $1.97 \mathrm{kcal} \mathrm{mol}^{-1}$. Changes in enthalpy $(\Delta H)$ range from $-2.06 \mathrm{kcal} \mathrm{mol}^{-1}$ (BCL2 3-7-1) to $-0.33 \mathrm{kcal} \mathrm{mol}^{-1}\left(\mathrm{hTel} 22 \mathrm{Na}^{+}\right)$differing by $1.73 \mathrm{kcal} \mathrm{mol}^{-1}$ for binding mode 3 . The entropic contribution $(-T \Delta S)$ ranges from $-7.01 \mathrm{kcal} \mathrm{mol}^{-1}\left(\mathrm{hTel22} \mathrm{Na}^{+}\right)$to $-4.07 \mathrm{kcal} \mathrm{mol}^{-1}$ (KRAS 6-4-6) yielding a difference of $2.94 \mathrm{kcal} \mathrm{mol}^{-1}$ for binding mode 3 .

Mixed parallel/antiparallel G4-DNAs KRAS 6-4-4, KRAS 6-45 , and BCL2 3-5-3 are driven by entropy with favorable enthalpic contributions. The long-looped parallel c-MYC 1-6-1 G4-DNA also shares this thermodynamic signature.

Parallel G4 BCL2 3-7-1 is driven primarily by enthalpy with a significant entropic contribution. Parallel G4 BCL2 3-6-2 is driven by enthalpy with a smaller, but favorable entropic contribution. The parallel short-looped c-MYC 1-2-1 shared this thermodynamic signature of a large enthalpic driving force with a smaller entropic contribution.
Mixed parallel/antiparallel G4-DNAs hTel22 $\left(\mathrm{K}^{+}\right)$and KRAS 6-4-6 were driven by enthalpy with a smaller, but still favorable entropic contribution.

Antiparallel G4-DNA hTel22 $\left(\mathrm{Na}^{+}\right)$was driven nearly entirely by enthalpy with a favorable entropic contribution smaller than $1 \mathrm{kcal} \mathrm{mol}^{-1}$.

The ITC binding experiments with several DNA foldamers, including c-MYC, KRAS, and duplex DNAs, were attempted for the hydroxysteroid dehydrogenase (HSD) series of analogs, though weak interactions and poor heat generation, even when utilizing reverse titrations, hindered the determination of the binding thermodynamics for most ligands. Forward titrations of HSD121 were able to generate preliminary binding data for G4DNAs c-MYC WT, c-MYC 1-6-1, KRAS 6-4-4, KRAS 5-4-5, and 25 bp duplex DNA. Other analogs in the series failed to generate meaningful binding curves, even using high concentrations of G4-DNA in a reverse titration experiment to circumvent the issues with ligand solubility. The use of DMSO was necessary to obtain useable concentrations for some of the DMZ analogs having lower solubility. Although we expected DMSO to have an influence on the binding parameters, these were screening experiments to determine if any of these compounds might have high affinity for G4-DNA. We had previously determined that binding trends observed in DMSO containing solutions having DMSO concentrations of $30 \%$ or less mimicked the relative affinities obtained in water for compounds having adequate solubility. If lower solubility DMZ analogs had been found that exhibited promising G4 affinity then we would explore methods to improve their solubility. Table S1 summarizes the thermodynamic parameters that were determined or an indication that the experiment was attempted. ITC thermograms of these exploratory experiments are found in the Supporting Information (Figures S8-S16).

CD. Parallel G4 c-MYC 1-6-1 demonstrates a slight increase in molar ellipticity in the $265 \mathrm{~nm}$ region upon addition of DMZ as seen in the CD data present in Figure 2. Parallel G4 c-MYC 12-1 demonstrates an attenuation in molar ellipticity in excess of 4 mol ratios DMZ.

Parallel quadruplex BCL2 3-6-2 and mixed parallel/ antiparallel quadruplexes BCL2 3-5-3, KRAS 6-4-4, and KRAS 6-4-6 resisted attenuation through the course of titration with DMZ. Mixed parallel/antiparallel quadruplex KRAS 6-4-5 demonstrated a slight attenuation in molar ellipticity, which was more pronounced in the $260 \mathrm{~nm}$ region than in the $290 \mathrm{~nm}$ region. Parallel quadruplex BCL2 3-7-1 demonstrated an attenuation in molar ellipticity at the 4:1 mol ratio DMZ/ DNA in the $265 \mathrm{~nm}$ region.

Antiparallel quadruplex hTel22 $\mathrm{Na}^{+}$showed a slight attenuation in molar ellipticity over the course of the titration by DMZ. Mixed parallel/antiparallel quadruplex hTel22 $\mathrm{K}^{+}$ resisted attenuation upon DMZ titration.

In most cases, the changes in $\mathrm{CD}$ signal are very small. The most important information that may be obtained from the $\mathrm{CD}$ data is that the target DNA is initially folded into a G4, and the final G4-DNA.DMZ complex is still folded into a G4.

BCL2 has been previously characterized by NMR, and the major G4 species formed adopts a mixed parallel/antiparallel topology with loop sizes 3,7 , and $1 .{ }^{24}$ More recently, parallel BCL2 G4s with 13 member loops have been reported. ${ }^{25}$ In the mutants generated for this work, the large loop bases have been mutated to adenine, which has different stacking inclinations than guanine in single-stranded DNA (as in the loops of a G4) and could be influencing the topologies characterized in this 


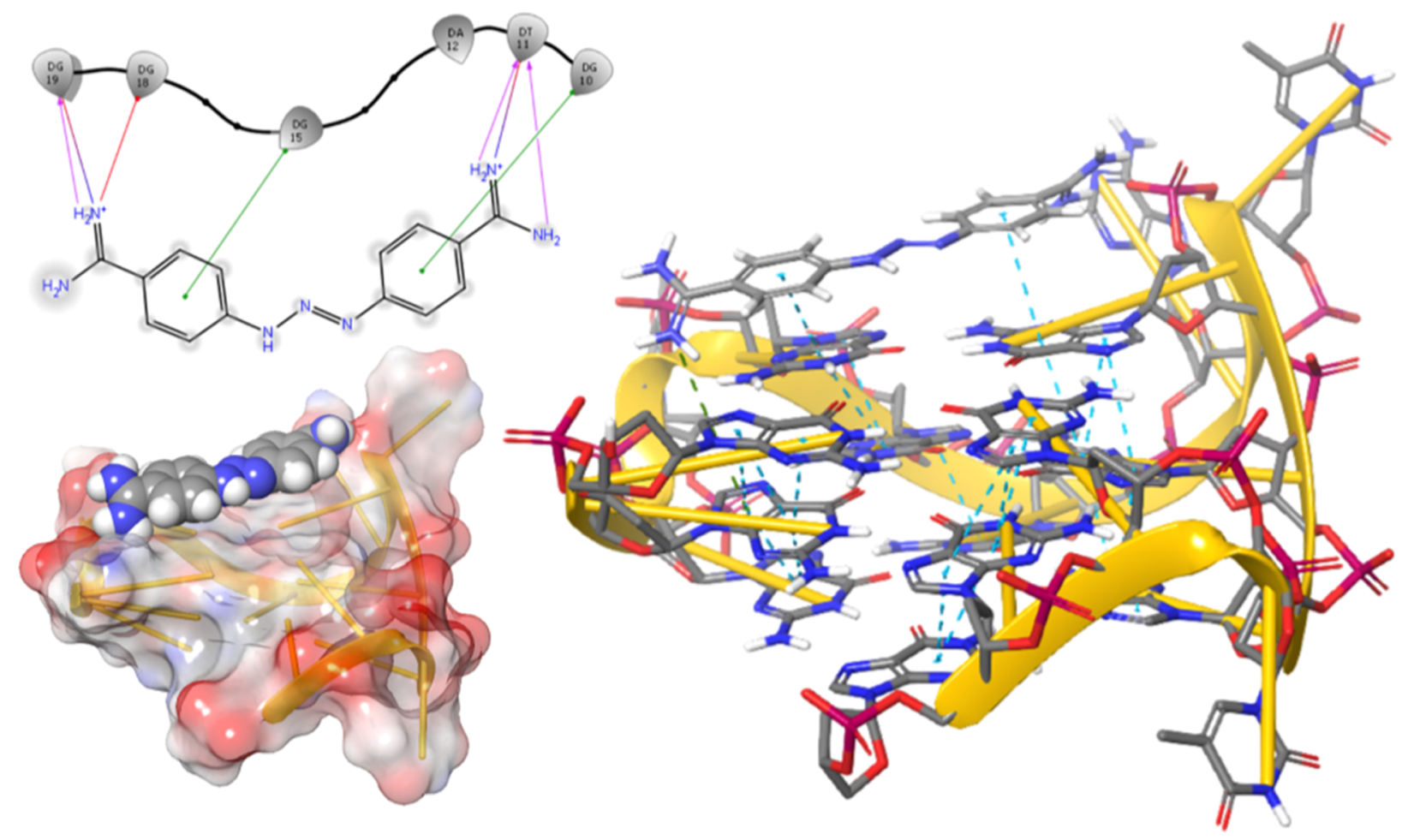

Figure 3. DMZ docked to c-MYC G4 $\left(\mathrm{PDB} 1 \mathrm{XAV}^{27}\right)$ in an end-stacked conformation. Ligand interactive diagram demonstrating $\pi$-stacking interactions with guanine 10 and 15 of model c-MYC G4 1XAV and DMZ (top left). Electrostatic potential colored surface applied to a cartoon representation of the c-MYC G4 with DMZ (Corey-Pauling-Koltun representation) interacting with the exposed tetrad face (bottom left). The $\pi$ stacking network of the c-MYC G4 continues through the bound DMZ molecule (right).

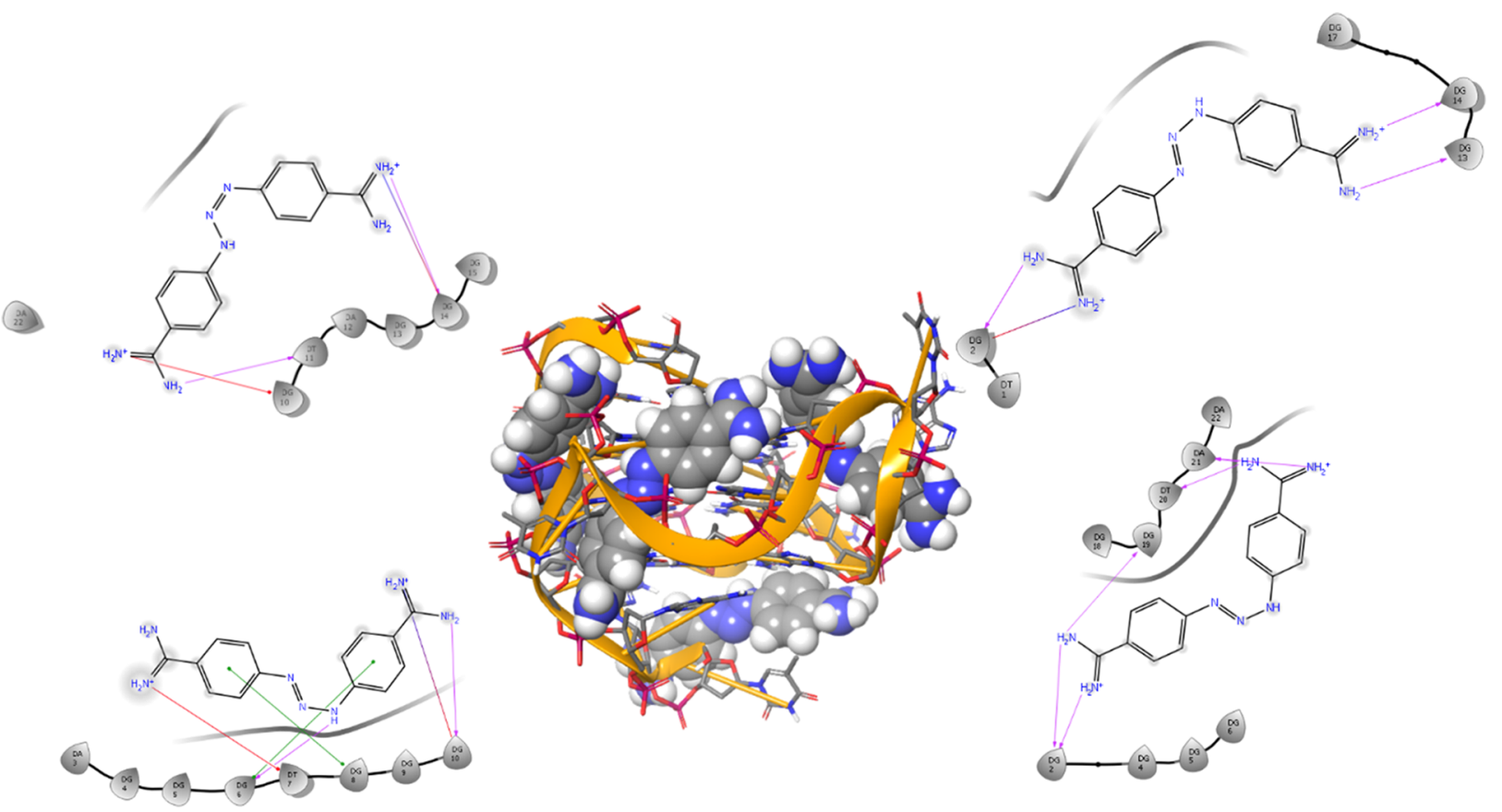

Figure 4. DMZ docked to c-MYC G4 (PDB $1 \mathrm{XAV}^{27}$ ) depicting loop interactions. The ligand interactive diagrams for the DMZ molecules are shown near the $\mathrm{CPK}$ representation of $\mathrm{DMZ}$ in the central model.

work. ${ }^{26}$ The CD spectra for mutants BCL2 3-6-2 and BCL2 3-7-

1 clearly show that a parallel G4 population is the major species present in solution, though the breadth of the BCL2 3-6-2 curve and a slight signal in the BCL2 3-7-1 $295 \mathrm{~nm}$ region is suggestive of a minor antiparallel containing species.
Modeling. Docking of DMZ to a model of the c-MYC G4 core produced several poses, including docking to the exposed tetrad face with the $\pi$-stacked network continued through the $\mathrm{DMZ}$ structure via bonding with the aligned aromatic rings and guanines 10 and 15 of the 1XAV crystal. H-Bonding interactions with the amidine groups were also observed, and both types of 
interactions are seen in the ligand interactive diagram present in Figure 3. DMZ was also observed to dock on the sides of the quadruplex, engaging in loop interactions, as seen in Figure 4.

ITC. The free energy for DMZ complexation with the G4s examined in this work demonstrate similar free energies for each binding mode ( $\Delta G$ within $\sim 2 \mathrm{kcal} \mathrm{mol}^{-1}$ for each mode). The individual contributions of enthalpy and entropy to each G4 mutant and binding mode varied by nearly $\sim 8.5 \mathrm{kcal} \mathrm{mol}^{-1}$ across G4-DNAs. Clearly, enthalpy/entropy compensation plays a role in maintaining an approximately constant Gibbs free energy in the binding of DMZ to G4-DNAs of varied sequences, lengths, and topologies.

c-MYC/DMZ Interactions. DMZ demonstrates a slightly stronger binding affinity for c-MYC 1-2-1 than c-MYC 1-6-1 (-11.37 and $-10.77 \mathrm{kcal} \mathrm{mol}^{-1}$ respectively). The binding profile of mode 1 differs greatly between each topology, with the short-looped c-MYC 1-2-1 mutant complexation with DMZ being driven by enthalpy $\left(-8.38 \mathrm{kcal} \mathrm{mol}^{-1}\right)$ and a smaller $\left(-2.97 \mathrm{kcal} \mathrm{mol}^{-1}\right)$ entropic contribution. Binding of DMZ to cMYC 1-6-1 is entropically driven $\left(-7.39 \mathrm{kcal} \mathrm{mol}^{-1}\right)$ with a smaller enthalpic contribution of $\left(-3.38 \mathrm{kcal} \mathrm{mol}^{-1}\right)$. Dehydration of the large loop of c-MYC 1-6-1 as it folds to cover DMZ ligands bound on the top face of the G-tetrad may account for the large entropic driving force and additional bonding character in the enthalpic term from the $\pi$-stacking interactions.

BCL2/DMZ Interactions. The thermodynamic signature describing the binding of DMZ to BCL2 G4s presented a range of values from -2 to $-9 \mathrm{kcal} \mathrm{mol}^{-1}$ for the enthalpic and entropic contributions to free energy.

BCL2 3-6-2 demonstrated the largest enthalpic contribution $\left(\Delta H=-8.77 \mathrm{kcal} \mathrm{mol}^{-1}\right)$ to the free energy $(\Delta G=-11.75 \mathrm{kcal}$ $\left.\mathrm{mol}^{-1}\right)$ and the smallest entropic contribution $(-T \Delta S=-2.98$ $\mathrm{kcal} \mathrm{mol}^{-1}$ ) for the mode 1 binding event. This mode 1 binding event was the tightest observed for the DNA construct examined in this study. The crystal structure of BCL2 $\left(2 \mathrm{~F} 8 \mathrm{U}^{24}\right)$ clearly shows a large loop covering one of the tetrad faces, so modulating this loops size would likely have a large impact on binding mode, as was observed in the ITC data collected for this work. The loop size of BCL2 3-6-2 may be a good fit for DMZ engaged in binding interaction directly in their vicinity. Potentially, DMZ binding to an exposed tetrad face engages with a loop to maximize constructive $\pi$-stacking. Restricted torsional freedom upon ligand binding has been discussed before $^{28}$ and it seem reasonable that the strong enthalpic binding component should lead to a larger degree of restricted torsional freedom, which leads to enthalpy/entropy compensation.

BCL2 3-7-1 demonstrated an enthalpic contribution $(\Delta H)$ of $-6.58 \mathrm{kcal} \mathrm{mol}^{-1}$ and an entropic contribution $(-T \Delta S)$ of $-4.78 \mathrm{kcal} \mathrm{mol}^{-1}$ resulting in the intermediate affinity mode 1 binding event for the BCL2 mutants examined $(\Delta G=-11.35$ $\mathrm{kcal} \mathrm{mol}^{-1}$ ). This longer second ( 7 base) loop likely provides space for DMZ to take advantage of the $\pi$-stacking interactions with the G-tetrad but may be too large for optimal contact. The one base loop may limit G4 relaxation to maximize $\pi$-stacking interactions, resulting in the lower enthalpic contribution observed. The larger entropic contribution is likely due to interactions with the long loop which may displace associated water molecules into bulk solvent.

BCL2 3-5-3 was the weakest binder of the BCL2 mutants $(\Delta G$ $\left.=-11.20 \mathrm{kcal} \mathrm{mol}^{-1}\right)$ was driven by entropy $(-T \Delta S=-9.15$

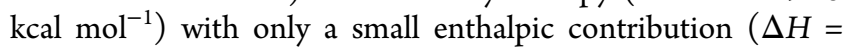

$-2.08 \mathrm{kcal} \mathrm{mol}^{-1}$ ). This was a mixed parallel/antiparallel G4 as determined by $\mathrm{CD}$ as opposed to the parallel G4 topology of the other BCL2 mutants. The small enthalpic term suggest that the G-tetrads may be less exposed than in the parallel mutants and interactions with the loops or sides of the G4 could be more favorable. The 5 and 3 membered loops may contribute to an overall poorer fit for making $\pi$-stacking contacts with the DMZ, resulting in the large entropy term for water release from desolvation of the DMZ/G4-DNA contact areas, but not extensive specific bonding character. The mixed topology may result in the loop not being able to fold onto the top of a G-tetrad as could be conceivable with the parallel BCL2 mutants based on their large enthalpic contributions, and may account for weaker enthalpic contributions for binding the BCL2 3-5-3 G4. It is also noteworthy that the mode 2 free energy of DMZ for BCL2 3-5-3 is approximately $0.5-1 \mathrm{kcal} \mathrm{mol}^{-1}$ weaker than the mode 2 free energy of BCL2 3-7-1 or BCL2 3-6-2, though the thermodynamic signatures are largely the same.

KRAS/DMZ Interactions. KRAS 6-4-4 demonstrated the largest free energy change $\left(\Delta G=-11.45 \mathrm{kcal} \mathrm{mol}^{-1}\right)$ upon DMZ binding of the KRAS mutants examined. This complexation was driven by entropy $\left(-T \Delta S=-7.16 \mathrm{kcal} \mathrm{mol}^{-1}\right)$ with a sizable enthalpic contribution $\left(\Delta H=-4.30 \mathrm{kcal} \mathrm{mol}^{-1}\right)$. This mutant likely possesses good accessibility to the G-tetrad faces and favorable interactions from the loops.

KRAS 6-4-6 showed the weakest binding mode 1 to DMZ with a free energy of $-10.3 \mathrm{kcal} \mathrm{mol}^{-1}$. Interestingly, when compared to the other KRAS mutants, this mutant's first binding mode demonstrated the largest enthalpic contribution $(\Delta H=$ $\left.-8.33 \mathrm{kcal} \mathrm{mol}^{-1}\right)$ and the smallest entropic term $(-T \Delta S=$ $\left.-2.02 \mathrm{kcal} \mathrm{mol}^{-1}\right)$. Perhaps the additional 6 membered loop is able to interact more favorably with the DMZ than the 5 or 4 membered loops of the other KRAS foldamers and better restrict the torsional freedom to reduce the favorable entropic contribution as postulated with BCL2 3-6-2.

KRAS 6-4-5 has a smaller enthalpic contribution $(\Delta H=$ $-3.41 \mathrm{kcal} \mathrm{mol}^{-1}$ ) than KRAS 6-4-4 with a similar entropic contribution $\left(-T \Delta S=-7.19 \mathrm{kcal} \mathrm{mol}^{-1}\right)$ resulting in a free energy of $\Delta G=-10.60 \mathrm{kcal} \mathrm{mol}^{-1}$. The loss of nearly $1 \mathrm{kcal}$ $\mathrm{mol}^{-1}$ from the enthalpy term due to the lengthening of the third loop may be the result of a poorer fit if the loop bends to cover DMZ bound to a tetrad face to generate $\pi$-bonding character. A $\mathrm{DMZ}$ molecule may alternatively have less favorable interactions with the loop engaging in binding interactions with the side of the G4.

hTel22/DMZ Interactions. The antiparallel hTel22 $\mathrm{Na}^{+} \mathrm{G} 4$ binds only slightly weaker than the mixed parallel/antiparallel hTel22 $\mathrm{K}^{+} \mathrm{G} 4\left(\Delta G=-11.2 \mathrm{vs}-11.07 \mathrm{kcal} \mathrm{mol}^{-1}\right)$ though the enthalpic terms vary by nearly $2 \mathrm{kcal} \mathrm{mol}^{-1}(\Delta H=-10.36$ and $-8.43 \mathrm{kcal} \mathrm{mol}^{-1}$ for hTel22 $\mathrm{Na}^{+}$and hTel2 $2 \mathrm{~K}^{+}$respectively). The entropic terms also vary by approximately $1.8 \mathrm{kcal} \mathrm{mol}^{-1}$ $\left(-T \Delta S=-0.81\right.$ and $-2.65 \mathrm{kcal} \mathrm{mol}^{-1}$ for hTel22 $\mathrm{Na}^{+}$and hTel22 $\mathrm{K}^{+}$respectively) The greater enthalpic driving force of the antiparallel G4 hTel22 $\mathrm{Na}^{+}$exhibited the most favorable enthalpic contribution $\left(\Delta H=-10.36 \mathrm{kcal} \mathrm{mol}^{-1}\right)$ and least favorable entropic contribution $\left(-T \Delta S=-0.81 \mathrm{kcal} \mathrm{mol}^{-1}\right)$ of the oligonucleotides examined. This purely antiparallel conformation possesses open top and bottom G-tetrads for binding of DMZ and the loops are 3 nucleotide bases long which potentially provides room for intercalation modes to be utilized. The mixed parallel/antiparallel hTel22 $\mathrm{K}^{+} \mathrm{G} 4$ has the open Gtetrad face and a very favorable enthalpic contribution to binding, but the larger entropic contribution of $-2.65 \mathrm{kcal} \mathrm{mol}^{-1}$ 
is required for the foldamers to display a similar binding affinity. This larger entropic contribution for the mixed parallel/ antiparallel species is likely a result of loop interactions with the DMZ.

$C D$. To determine the structural effects of the interactions between the DMZ and G4-DNAs, we performed a series of CD titration experiments in which DMZ was added to the various G4s. CD titrations were performed in the $\mathrm{K}^{+}$tris buffer containing 30\% DMSO. Under these experimental conditions, the free c-MYC, KRAS, and BCL2 G4s exhibited characteristic $\mathrm{CD}$ spectral features that are similar to those previously published in the literature. ${ }^{29}$ It is well known that putative G4 forming sequences typically yield an ensemble of G4 isomers with different topologies. This is the reason that we used mutant (e.g., 1-2-1 and 1-6-1) sequences with restricted folded conformations. The use of CD in these studies was (1) to confirm the existence of G4 motifs, (2) to determine any preference DMZ compounds might have for a particular foldamer, and (3) to determine if binding DMZ (or analogs) would perturb the folding equilibria in the ensemble.

Upon complexation with DMZ, the CD signals for c-MYC 12-1 and BCL2 3-7-1 dramatically attenuated. These G4-DNAs demonstrate an attenuation in molar ellipticity in excess of $4 \mathrm{~mol}$ ratios DMZ. This could be interpreted as consistent with 2 molecules of DMZ binding to each exposed tetrad face (top and bottom) of the G4 before a loop interactive mode is utilized, which may disrupt the G4 motif structure. A plausible explanation for this is that the inherent G-tetrad $\pi-\pi$ stacking interactions are weakened upon DMZ binding. Since both of these G4-DNAs have a loop containing a single base, there may not be sufficient space for the G4 to relax and allow the insertion of DMZ into the loops without the distortion of the G4 core. The other mutant with single bases in loops, c-MYC 1-6-1 demonstrates a slight increase in molar ellipticity in the $265 \mathrm{~nm}$ region. This may result from an increased propensity for the extended loop to fold over the tetrad in the presence of ligand.

The G4-DNA hTel22 $\mathrm{Na}^{+}$demonstrates a slight decrease in molar ellipticity upon titration with DMZ. This is inconsistent with the conservation of signal in the $290 \mathrm{~nm}$ region previously observed as constant upon DMZ titration, but the buffer in this case has been supplemented with DMSO and contain a higher salt concentration (previously $100 \mathrm{mM} \mathrm{NaCl}$ ), which may explain the difference. ${ }^{23}$

The G4-DNA hTel22 $\mathrm{K}^{+}$resisted a change in molar ellipticity, indicating that the structure was largely unchanged as a result of DMZ binding.

KRAS 6-4-5 demonstrated an attenuation in molar ellipticity, which was more pronounced in the $260 \mathrm{~nm}$ region than in the $290 \mathrm{~nm}$ region. This is consistent with a slight decrease in quadruplex structure, possibly the result of a preferential perturbation of the parallel G4 population.

The remaining G4s do not demonstrate attenuation. It is possible that the larger loop sizes provide flexibility such that DMZ binding does not cause a deformation of the stacked Gtetrads and the $\pi-\pi$ binding network. The lateral loops may allow the G4 to relax and unwind slightly to accommodate the DMZ.

There was no evidence for an induce ligand CD signal when DMZ was bound to the different G4s.

Representative CD experiments with the analog series are shown in Figures S5-S7 and generally show only small changes in molar ellipticity, which are most likely the result of small errors in the baseline or instrument drift. In fact, DMSO results in such large disruption in the region below $240 \mathrm{~nm}$, that region has been removed from the CD figures. In effect, the large DMSO signal in the shorter wavelength region precludes accurate measurement of G4 ellipticities below about $250 \mathrm{~nm}$. As with the DMZ titrations, it seems the most important information that is obtained from the CD spectra is that the target G4-DNA is present in the folded state before and after the titration of the analogs.

Modeling. The stoichiometry of DMZ interacting with a cMYC G4 has been determined previously via ESI-MS to be eight DMZ molecules per G4. ${ }^{23}$ The modeling data demonstrate a rational for the reported binding, whereby two molecules of DMZ may interact with both the top and bottom of the G4 and four DMZ molecules interaction with the sides, for a total of eight.

An alternative model for the binding would be the relaxation of the G4 as DMZ intercalated, as has been a reported binding mode of TMPYP $4 .{ }^{30}$ Figure 3 demonstrates how the $\pi$-stacking network is not interrupted by the binding of DMZ, and one may visualize $2 \mathrm{DMZ}$ molecules oriented between G-tetrads as able to line up with the guanine molecules and engage the network. As many as four DMZ molecules would intercalate into the G4 if this binding mode utilized with occupancy at the ends as well, also for a total of eight. The relatively weak enthalpic contributions of binding modes 2 and 3 for the G4s examined reveal that entropy tends to be the driving force (see Figures S1-S3 for thermodynamic profiles). Though intercalation would require a near complete stripping of water from the ligand, which would result in a strong entropic contribution due to increased torsional freedom of the solvent, it would be expected that $\pi$-stacking interactions would result in very favorable enthalpic term, which was not observed experimentally.

\section{CONCLUSIONS}

The present work characterized the complexation of 1 antiparallel, 4 parallel, and 5 mixed parallel/antiparallel G4 forming oligonucleotides forming complexes with DMZ. Though the free energy of the strongest binding event varied by only $\sim 1.5 \mathrm{kcal} \mathrm{mol}^{-1}$ among the oligonucleotides examined, the thermodynamic profile varied greatly, with the observation of enthalpy contributions ranging from -10.36 to $-2.08 \mathrm{kcal}$ $\mathrm{mol}^{-1}$ (difference of $8.28 \mathrm{kcal} \mathrm{mol}^{-1}$ ) and entropic contributions from -9.15 to $-0.81 \mathrm{kcal} \mathrm{mol}^{-1}$ (difference of $8.34 \mathrm{kcal} \mathrm{mol}^{-1}$ ). Enthalpy/entropy compensation is clearly a large factor in the binding of DMZ to G4 motifs, and the selective modification of $\mathrm{DMZ}$ to improve either the entropic or enthalpic binding character may lead to enhanced G4 selectivity.

\section{EXPERIMENTAL SECTION}

ITC experiments were performed using a Microcal VP-ITC (Malvern Panalytical) at $25{ }^{\circ} \mathrm{C}$ in which a typical experiment involved injection of 35 aliquots of dilute ligand ( 4000) $\mu \mathrm{M}$ into the calorimeter cell containing $1.45 \mathrm{~mL}$ of $\sim 10 \mu \mathrm{M}$ DNA. Injection volumes were typically $8 \mu \mathrm{L}$, and injections were made at $250 \mathrm{~s}$ intervals. The reverse titration was also utilized owing to the low solubility of several ligands under examination. A typical reverse titration experiment involved injecting aliquots of stock $0.6311 \mathrm{mM}$ G4-DNA into the calorimeter cell containing 1.45 $\mathrm{mL}$ of $30 \mu \mathrm{M}$ ligand. Reverse titration injection volumes were 2 $\mu \mathrm{L}$, and injections were made at $200 \mathrm{~s}$ intervals. The ITC thermograms were corrected for titrate and titrant dilution 
effects by performing the appropriate blank experiments and correcting the observed heats by subtracting the heats of dilution. Data analysis was performed using CHASM, an in house data analysis program developed by the Lewis Lab to perform nonlinear regression fits of the corrected ITC data. ${ }^{31}$

CD experiments were performed using an Olis DSM-20 spectropolarimeter (Bogart, GA). Typical experiments involved the titration of a DNA sample with a nominal $A_{260}=1.0$ with ligand sample at $25{ }^{\circ} \mathrm{C}$. Corrections were made by subtracting the signal of ligand titrated into buffer from its respective sample and converting to molar ellipticity.

Computational analysis of the c-MYC binding modes of DMZ was performed using Maestro Version 11.3.016 by Schrodinger. $\mathrm{PDB}$ entry $1 \mathrm{XAV}^{27}$ was imported into Maestro, and the "Protein Preparation Wizard" was used to preprocess and minimize the structure using the OPLS3 force field. The "SiteMap" utility was used to identify potential binding sites, which were used as targets for the Receptor Grid Generation tool to create grids to define potential docking sites, and a grid was placed at the face of the G-tetrad. Docking to the end of the c-MYC G4 core required truncation of the DNA strand to remove residues covering the G-tetrad, which would be inflexible during computational docking, but dynamic in solution. DMZ was drawn using the internal build functions and subjected to the "LigPrep" utility to prepare for docking. The "Ligand Docking" tool was used at the XP (extra precision) level.

\section{MATERIALS}

Target DNAs were c-MYC, KRAS, and BCL2 G4 forming mutants with restricted folding topologies purchased from Midland Certified Reagent Company. The G4 forming DNA was purchased as the RP grade and used without further purification. The sequences are as follows: c-MYC 1-6-1: 5'TGG GGA GGG TTT TTA GGG TGG GGA-3'; c-MYC 1-21: 5' -TTT TTA GGG TGG GGA GGG TGG GGA-3'; BCL2 37-1: 5'-CGG GCA CGG GAA AAA AAG GGC GGG AAC-3'; BCL2 3-6-2: 5' -CGG GCA CGG GAA AAA AGG GAC GGG AAC-3'; BCL2 3-5-3: 5'-CGG GCA CGG GAA AAA GGG AAC GGG AAC-3'; KRAS 6-4-4: 5'-GGG CAA TAT GGG AAA AGG GAA AAG GGA AAA-3'; KRAS 6-4-5: 5'-GGG CAA TAT GGG AAA AGG GAA AAA GGG AAA-3'; KRAS 64-6: 5'-GGG CAA TAT GGG AAA AGG GAA AAA AGG GAA-3' hTel22: 5'-AGG GTT AGG GTT AGG GTTA GGG$3^{\prime}$; 25 bp duplex and its complementary strand: 5'-ACT TGC TCA CAG ACC GTA GCT TGA T-3' \& 5'-ATC AAG CTA CGG TAT GTG AGC AAG T-3', respectively.

The DNA was exhaustively dialyzed against $\mathrm{K}^{+}$tris buffer $(150$ $\mathrm{mM} \mathrm{KCl}, 20 \mathrm{mM}$ tris, $1 \mathrm{mM}$ EDTA, 30\% DMSO, pH 7.2) (or $\mathrm{Na}^{+}$tris buffer containing $150 \mathrm{mM} \mathrm{NaCl}$ in place of $\mathrm{KCl}$ for hTel22 $\mathrm{Na}^{+}$) and annealed by heating to $95{ }^{\circ} \mathrm{C}$ and holding for 10 min before cooling to $5{ }^{\circ} \mathrm{C}$ at a rate of $0.5^{\circ} \mathrm{C} \mathrm{min}{ }^{-1}$. DNAs were likewise prepared in $\mathrm{K}^{+}$acetate buffer $\left(150 \mathrm{mM} \mathrm{K}^{+}, 20 \mathrm{mM}\right.$ acetate, $1 \mathrm{mM}$ EDTA, 30\% DMSO, pH 5.0), which was necessary to improve solubility of the DMZ analogs to a point where their concentration would be sufficient for meaningful heat generation in the ITC. DNA stock concentrations were verified using the following extinction coefficients provided by Midland Certified Reagent Company: c-MYC 1-6-1 $\varepsilon_{260}=270$ $300 \mu \mathrm{M}^{-1} \mathrm{~cm}^{-1}$; c-MYC 1-2-1 $\varepsilon_{260}=270300 \mu \mathrm{M}^{-1} \mathrm{~cm}^{-1}$; BCL2 3-7-1 $\varepsilon_{260}=329400 \mu \mathrm{M}^{-1} \mathrm{~cm}^{-1}$; BCL2 3-6-2 $\varepsilon_{260}=329400$ $\mu \mathrm{M}^{-1} \mathrm{~cm}^{-1}$; BCL2 3-5-3 $\varepsilon_{260}=329400 \mu \mathrm{M}^{-1} \mathrm{~cm}^{-1}$; KRAS 6-4$4 \varepsilon_{260}=393200 \mu \mathrm{M}^{-1} \mathrm{~cm}^{-1}$; KRAS 6-4-5 $\varepsilon_{260}=393200 \mu \mathrm{M}^{-1}$ $\mathrm{cm}^{-1}$; KRAS 6-4-6 $\varepsilon_{260}=393200 \mu \mathrm{M}^{-1} \mathrm{~cm}^{-1}$; hTel22 $\varepsilon_{260}=$ $253400 \mu \mathrm{M}^{-1} \mathrm{~cm}^{-1}$; 25 bp duplex $\varepsilon_{260}=397000 \mu \mathrm{M}^{-1} \mathrm{~cm}^{-1}$.

DMZ was purchased from Santa Cruz Biotechnology, Inc. and dissolved in the final dialysate buffer using heat and sonication to assist in dissolution. DMZ concentration was verified using an extinction coefficient of $\varepsilon_{370}=36700 \mu \mathrm{M}^{-1} \mathrm{~cm}^{-1}$.

DMZ analog synthesis is described in the Supporting Information. Concentrations were verified using experimentally determined extinction coefficients. ITC experiments were performed with the analog concentration below the experimentally determined limit of solubility in each buffer system.

\section{ASSOCIATED CONTENT}

\section{Supporting Information}

The Supporting Information is available free of charge on the ACS Publications website at DOI: 10.1021/acsomega.8b01621.

Results of exploratory DMZ analog binding to DNA; thermodynamic profile of binding modes for DMZ/G4DNA interaction; DMZ analog structures; CD scans of DMZ analogs with selected BCL 2 and KRAS mutant G4s; raw thermograms for DMZ analogs with G4-DNAs; supplemental experimental procedures; ${ }^{1} \mathrm{H}$ and ${ }^{13} \mathrm{C}$ NMR spectra (PDF)

\section{AUTHOR INFORMATION}

\section{Corresponding Authors}

*E-mail: hsintim@purdue.edu (H.O.S.).

*E-mail: elewis@chemistry.edu (E.A.L.).

ORCID

Clinton G. Mikek: 0000-0001-5662-3096

Edwin A. Lewis: 0000-0003-4432-1482

Notes

The authors declare no competing financial interest.

\section{ACKNOWLEDGMENTS}

NSF Award\# CHE 1518006.

\section{REFERENCES}

(1) (a) Gellert, M.; Lipsett, M. N.; Davies, D. R. Helix formation by guanylic acid. Proc. Natl. Acad. Sci. U.S.A. 1962, 48, 2013-2018. (b) Bochman, M. L.; Paeschke, K.; Zakian, V. A. DNA secondary structures: stability and function of G-quadruplex structures. Nat. Rev. Genet. 2012, 13, 770-780. (c) Kumari, S.; Bugaut, A.; Huppert, J. L.; Balasubramanian, S. An RNA G-quadruplex in the $5^{\prime}$ UTR of the NRAS proto-oncogene modulates translation. Nat. Chem. Biol. 2007, 3, 218221

(2) Sklenár, V.; Feigon, J. Formation of a stable triplex from a single DNA strand. Nature 1990, 345, 836-838.

(3) (a) Brooks, T. A.; Kendrick, S.; Hurley, L. Making sense of Gquadruplex and i-motif functions in oncogene promoters. FEBS J. 2010, 277, 3459-3469. (b) Gehring, K.; Leroy, J. L.; Gueron, M. A tetrameric DNA structure with protonated cytosine.cytosine base pairs. Nature 1993, 363, 561-565.

(4) (a) Balasubramanian, S.; Hurley, L. H.; Neidle, S. Targeting Gquadruplexes in gene promoters: a novel anticancer strategy? Nat. Rev. Drug Discovery 2011, 10, 261-275. (b) Düchler, M. G-quadruplexes: targets and tools in anticancer drug design. J. Drug Targeting 2012, 20, 389-400. (c) Collie, G. W.; Parkinson, G. N. The application of DNA and RNA G-quadruplexes to therapeutic medicines. Chem. Soc. Rev. 2011, 40, 5867-5892.

(5) (a) Huppert, J. L.; Balasubramanian, S. Prevalence of quadruplexes in the human genome. Nucleic Acids Res. 2005, 33, 2908-2916. (b) Todd, A. K.; Johnston, M.; Neidle, S. Highly prevalent putative 
quadruplex sequence motifs in human DNA. Nucleic Acids Res. 2005, 33, 2901-2907.

(6) Xu, Y. Chemistry in human telomere biology: structure, function and targeting of telomere DNA/RNA. Chem. Soc. Rev. 2011, 40, 27192740.

(7) Mathad, R. I.; Hatzakis, E.; Dai, J.; Yang, D. c-MYC promoter Gquadruplex formed at the $5^{\prime}$-end of NHE $\mathrm{III}_{1}$ element: insights into biological relevance and parallel-stranded G-quadruplex stability. Nucleic Acids Res. 2011, 39, 9023-9033.

(8) Cogoi, S.; Xodo, L. E. G-quadruplex formation within the promoter of the KRAS proto-oncogene and its effect on transcription. Nucleic Acids Res. 2006, 34, 2536-2549.

(9) Dai, J.; Dexheimer, T. S.; Chen, D.; Carver, M.; Ambrus, A.; Jones, R. A.; Yang, D. An intramolecular G-quadruplex structure with mixed parallel/antiparallel G-strands formed in the human BCL-2 promoter region in solution. J. Am. Chem. Soc. 2006, 128, 1096-1098.

(10) Phan, A. T.; Kuryavyi, V.; Gaw, H. Y.; Patel, D. J. Small-molecule interaction with a five-guanine-tract g-quadruplex structure from the human myc promoter. Nat. Chem. Biol. 2005, 1, 167-173.

(11) Bejugam, M.; Sewitz, S.; Shirude, P. S.; Rodriguez, R.; Shahid, R.; Balasubramanian, $\mathrm{S}$. Trisubstituted Isoalloxazines as a New Class of GQuadruplex Binding Ligands: Small Molecule Regulation of c-kit Oncogene Expression. J. Am. Chem. Soc. 2007, 129, 12926-12927.

(12) (a) Balasubramanian, S.; Hurley, L. H.; Neidle, S. Targeting Gquadruplexes in gene promoters: a novel anticancer strategy? Nat. Rev. Drug Discovery 201 1, 10, 261-275. (b) Tera, M.; Ishizuka, H.; Takagi, M.; Suganuma, M.; Shin-ya, K.; Nagasawa, K. Macrocyclic Hexaoxazoles as Sequence- and Mode-Selective G-Quadruplex Binders. Angew. Chem., Int. Ed. 2008, 47, 5557-5560. (c) Gomez, D.; Lemarteleur, T.; Lacroix, L.; Mailliet, P.; Mergny, J. L.; Riou, J. F. Telomerase downregulation induced by the G-quadruplex ligand 12459 in A549 cells is mediated by hTERT RNA alternative splicing. Nucleic Acids Res. 2004, 32, 371-379.

(13) Chen, M. C.; Murat, P.; Abecassis, K.; Ferre-D’Amare, A. R.; Balasubramanian, S. Insights into the mechanism of a G-quadruplexunwinding DEAH-box helicase. Nucleic Acids Res. 2015, 43, 22232231.

(14) (a) Martínez, R.; Chacon-Garcia, L. The Search of DNAIntercalators as Antitumoral Drugs: What it Worked and What did not Work. Curr. Med. Chem. 2005, 12, 127-151. (b) Le, T. V. T.; Sujin, H.; Junghyun, C.; Hyun-Ju, P. G-Quadruplex Binding Ligands: from Naturally Occurring to Rationally Designed Molecules. Curr. Pharm. Des. 2012, 18, 1948-1972. (c) Alzeer, J.; Vummidi, B. R.; Roth, P. J.; Luedtke, N. W. Guanidinium-modified phthalocyanines as high-affinity G-quadruplex fluorescent probes and transcriptional regulators. Angew. Chem., Int. Ed. 2009, 48, 9362-9365. (d) Ou, T. M.; Lu, Y. J.; Tan, J. H.; Huang, Z. S.; Wong, K. Y.; Gu, L. Q. G-quadruplexes: targets in anticancer drug design. ChemMedChem 2008, 3, 690-713.

(15) Sundquist, W. I.; Heaphy, S. Evidence for interstrand quadruplex formation in the dimerization of human immunodeficiency virus 1 genomic RNA. Proc. Natl. Acad. Sci. U.S.A. 1993, 90, 3393-3397.

(16) Marchetti, C.; Zyner, K. G.; Ohnmacht, S. A.; Robson, M.; Haider, S. M.; Morton, J. P.; Marsico, G.; Vo, T.; Laughlin-Toth, S.; Ahmed, A. A.; Di Vita, G.; Pazitna, I.; Gunaratnam, M.; Besser, R. J.; Andrade, A. C. G.; Diocou, S.; Pike, J. A.; Tannahill, D.; Pedley, R. B.; Evans, T. R. J.; Wilson, W. D.; Balasubramanian, S.; Neidle, S. Targeting Multiple Effector Pathways in Pancreatic Ductal Adenocarcinoma with a G-Quadruplex-Binding Small Molecule. J. Med. Chem. 2018, 61, 2500-2517.

(17) Ou, A.; Guédin, A.; Skelton, B. W.; Amrane, S.; Evans, C. W.; Norret, M.; Iyer, S.; Mergny, J.-L.; Smith, N. M. Multicarbazole scaffolds for selective G-quadruplex binding. Chem. Commun. 2018, 9647.

(18) Bouzada, D.; Salvadó, I.; Barka, G.; Rama, G.; Martínez-Costas, J.; Lorca, R.; Somoza, Á.; Melle-Franco, M.; Vázquez, M. E.; Vázquez López, M. Selective G-quadruplex binding by oligoarginine-Ru(dppz) metallopeptides. Chem. Commun. 2018, 54, 658-661.

(19) Lin, Y.-H.; Chuang, S.-M.; Wu, P.-C.; Chen, C.-L.; Jeyachandran, S.; Lo, S.-C.; Huang, H.-S.; Hou, M.-H. Selective recognition and stabilization of new ligands targeting the potassium form of the human telomeric G-quadruplex DNA. Sci. Rep. 2016, 6, No. 31019.

(20) Franceschin, M.; Cianni, L.; Pitorri, M.; Micheli, E.; Cacchione, S.; Frezza, C.; Serafini, M.; Hu, M.-H.; Su, H.; Huang, Z.; Gu, L.; Bianco, A. Natural Aromatic Compounds as Scaffolds to Develop Selective G-Quadruplex Ligands: From Previously Reported Berberine Derivatives to New Palmatine Analogues. Molecules 2018, 23, No. 1423.

(21) Miller, K. M.; Rodriguez, R. G-quadruplexes: selective DNA targeting for cancer therapeutics? Expert Rev. Clin. Pharmacol. 2011, 4, 139-142.

(22) Wang, C.; Carter-Cooper, B.; Du, Y.; Zhou, J.; Saeed, M. A.; Liu, J.; Guo, M.; Roembke, B.; Mikek, C.; Lewis, E. A.; Lapidus, R. G.; Sintim, H. O. Alkyne-substituted diminazene as G-quadruplex binders with anticancer activities. Eur. J. Med. Chem. 2016, 118, 266-275.

(23) Zhou, J.; Le, V.; Kalia, D.; Nakayama, S.; Mikek, C.; Lewis, E. A.; Sintim, H. O. Diminazene or berenil, a classic duplex minor groove binder, binds to G-quadruplexes with low nanomolar dissociation constants and the amidine groups are also critical for G-quadruplex binding. Mol. BioSyst. 2014, 10, 2724-2734.

(24) Dai, J.; Chen, D.; Jones, R. A.; Hurley, L. H.; Yang, D. NMR solution structure of the major G-quadruplex structure formed in the human BCL2 promoter region. Nucleic Acids Res. 2006, 34, 5133-5144.

(25) Agrawal, P.; Lin, C.; Mathad, R. I.; Carver, M.; Yang, D. The Major G-Quadruplex Formed in the Human BCL-2 Proximal Promoter Adopts a Parallel Structure with a 13-nt Loop in K+ Solution. J. Am. Chem. Soc. 2014, 136, 1750-1753.

(26) McIntosh, D. B.; Duggan, G.; Gouil, Q.; Saleh, O. A. SequenceDependent Elasticity and Electrostatics of Single-Stranded DNA: Signatures of Base-Stacking. Biophys. J. 2014, 106, 659-666.

(27) Ambrus, A.; Chen, D.; Dai, J.; Jones, R. A.; Yang, D. Solution Structure of the Biologically Relevant G-Quadruplex Element in the Human c-MYC Promoter. Implications for G-Quadruplex Stabilization. Biochemistry 2005, 44, 2048-2058.

(28) Chang, C.-e. A.; Chen, W.; Gilson, M. K. Ligand configurational entropy and protein binding. Proc. Natl. Acad. Sci. U.S.A. 2007, 104, 1534-1539.

(29) (a) Hudson, J. S.; Ding, L.; Le, V.; Lewis, E.; Graves, D. Recognition and binding of human telomeric G-quadruplex DNA by unfolding protein 1. Biochemistry 2014, 53, 3347-3356. (b) Dettler, J. M.; Buscaglia, R.; Le, V. H.; Lewis, E. A. DSC deconvolution of the structural complexity of c-MYC P1 promoter G-quadruplexes. Biophys. J. 2011, 100, 1517-1525. (c) Nagesh, N.; Buscaglia, R.; Dettler, J. M.; Lewis, E. A. Studies on the site and mode of TMPyP4 interactions with Bcl-2 promoter sequence G-Quadruplexes. Biophys. J. 2010, 98, 26282633. (d) Le, V. H.; Nagesh, N.; Lewis, E. A. Bcl-2 Promoter Sequence G-Quadruplex Interactions with Three Planar and Non-Planar Cationic Porphyrins: TMPyP4, TMPyP3, and TMPyP2. PLoS One 2013, 8, No. e72462. (e) Lavrado, J.; Brito, H.; Borralho, P. M.; Ohnmacht, S. A.; Kim, N.-S.; Leitão, C.; Pisco, S.; Gunaratnam, M.; Rodrigues, C. M. P.; Moreira, R.; Neidle, S.; Paulo, A. KRAS oncogene repression in colon cancer cell lines by G-quadruplex binding indolo[3,2-c]quinolines. Sci. Rep. 2015, 5, No. 9696.

(30) Cashman, D. J.; Buscaglia, R.; Freyer, M. W.; Dettler, J.; Hurley, L. H.; Lewis, E. A. Molecular modeling and biophysical analysis of the cMYC NHE-III 1 silencer element. J. Mol. Model. 2008, 14, 93-101.

(31) Le, V. H.; Buscaglia, R.; Chaires, J. B.; Lewis, E. A. Modeling complex equilibria in isothermal titration calorimetry experiments: thermodynamic parameters estimation for a three-binding-site model. Anal. Biochem. 2013, 434, 233-241. 